Eur. J. Clin. Chem. Clin. Biochem.

Vol. 32, 1994, pp. 543-547

(c) 1994 Walter de Gruyter \& Co.

Berlin $\cdot$ New York

\title{
Glycogen Phosphorylase Isoenzyme BB Mass Release After Coronary Artery Bypass Grafting
}

\author{
By P. Mair ${ }^{1}$, J. Mair ${ }^{2}$, E.-G. Krause ${ }^{3}$, D. Balogh ${ }^{1}$, B. Puschendorf ${ }^{2}$ and G. Rabitzsch ${ }^{3}$
}

${ }^{1}$ Klinik für Anästhesie und Intensivmedizin, Universität Innsbruck, Innsbruck, Austria

2 Institut für Medizinische Chemie und Biochemie, Universität Innsbruck, Innsbruck, Austria

3 Forschungsschwerpunkt Kardiologie, Max Delbrück Centrum für Molekulare Medizin, Berlin-Buch, Germany

(Received January 24/April 5, 1994)

Summary: Glycogen phosphorylase isoenzyme BB mass release was studied in 20 patients undergoing coronary artery bypass grafting. In 16 patients with uneventful coronary artery bypass grafting, glycogen phosphorylase isoenzyme BB mass concentrations showed a significant, transient increase in the post cross-clamping period and decreased to baseline values within 20 hours (peak concentrations ranged from $12.7 \mu \mathrm{g} / 1$ to $47.5 \mu \mathrm{g} / \mathrm{l}$, median 40 $\mu \mathrm{g} / \mathrm{l})$. One patient did not fulfil criteria for perioperative myocardial infarction, but clinical data indicated myocardial injury after aortic unclamping. In this patient only glycogen phosphorylase isoenzyme BB mass concentration and not creatine kinase isoenzyme MB catalytic concentration was increased, compared with uneventful patients. In 2 patients with emergency coronary artery bypass grafting for evolving myocardial infarction, glycogen phosphorylase isoenzyme $\mathrm{BB}$ mass concentrations, but not creatine kinase isoenzyme $\mathrm{MB}$ catalytic concentrations, correlated with clinical evidence of myocardial ischaemia. Our data indicate that glycogen phosphorylase isoenzyme BB mass concentration is a very sensitive laboratory marker of perioperative myocardial injury in patients undergoing coronary artery bypass grafting.

\section{Introduction}

Glycogen phosphorylase ${ }^{1}$ ) is the key enzyme of glycogenolysis. It exists as 3 different isoenzymes: $\overline{\mathrm{BB}}$ (brain), MM (muscle) and LL (liver). The isoenzyme BB of glycogen phosphorylase is also found in human myocardium, where it is the predominant isotype. Skeletal muscle, by contrast, contains only isoenzyme MM (1). Glycogenolysis is strongly accelerated in ischaemic myocardium, and ischaemic myocardium releases significant amounts of glycogen phosphorylase isoenzyme $\mathrm{BB}$ into the systemic circulation $(2,3,4)$. Increased glycogen phosphorylase isoenzyme BB mass concentrations are a very early laboratory marker of myocardial infarction in non-surgical patients (5). The aim of this

\footnotetext{
1) Enzymes:

Creatine kinase, EC 2.7.3.2.

Glycogen phosphorylase EC 2.4.1.1.
}

study was to investigate glycogen phosphorylase isoenzyme BB release in patients undergoing coronary artery bypass grafting, in an attempt to elucidate the role of glycogen phosphorylase isoenzyme $\mathrm{BB}$ mass concentration in the laboratory diagnosis of perioperative myocardial injury in bypass surgery.

\section{Methods}

Study subjects

After institutional approval and informed consent 20 consecutive patients ( 17 men, 3 women) undergoing coronary artery bypass grafting were investigated. The median age was 60.5 years (range: 45-76 years). Eighteen patients underwent elective coronary artery bypass grafting, the remaining 2 patients emergency coronary artery bypass grafting for evolving myocardial infarction. Fifteen of the 20 patients had a history of previous myocardial infarction. In 4 patients the preoperative left ventricular ejection fraction was below $45 \%$. Indications for surgical revascularisation were left main coronary artery stenosis in 7 and three vessel disease in 13 patients. On average, 3.6 grafts (range: $2-5$ ) per patient were im- 
planted. The internal mammary artery was used as a bypass vessel in 11 patients. Perioperative myocardial infarction was diagnosed when creatine ${ }^{l}$ ) kinase isoenzyme MB catalytic concentration exceeded 50 U/l 20 hours after aortic unclamping (6) and electrocardiography indicated new myocardial ischaemia. Serial venous blood samples were collected before surgery, before cardiopulmonary bypass, with aortic unclamping, $1,2,3,4,8,12,16,20,24$, 36 and 48 hours later.

Standard cardiopulmonary bypass technique with moderate systemic hypothermia and aortic crossclamping was used in all patients. Median aortic crossclamping time was 55 minutes (range: 36-83 minutes). Myocardial protection during aortic crossclamping was achieved by infusion of cold cristalloid cardioplegic solution (St Thomas solution) into the aortic root and by additional topical cooling.

\section{Laboratory analysis}

Creatine kinase and creatine kinase isoenzyme $M B$ catalytic concentrations were measured at $25^{\circ} \mathrm{C}$ with a Merck test kit (Darmstadt, Germany). Creatine kinase isoenzyme MB was measured by immunoinhibition. The upper limits of the reference interval of creatine kinase in non-surgical patients are $70 \mathrm{U} / \mathrm{l}$ for women and $80 \mathrm{U} / \mathrm{l}$ for men. The normal range of creatine kinase isoenzyme MB catalytic concentration is below $10 \mathrm{U} / \mathrm{l}$. Glycogen phosphorylase isoenzyme BB mass concentrations were determined by a recently developed sensitive immunoenzymometric assay, which is based on 2 monoclonal antibodies that are highly specific for glycogen phosphorylase isoenzyme $\mathrm{BB}$ (7). The median glycogen phosphorylase isoenzyme $\mathrm{BB}$ plasma concentration in a control population with chronic stable coronary artery disease $(21$ male, 10 female, median age 66 years) was $3.9 \mu \mathrm{g} / \mathrm{l}$ (range: $1.1 \mu \mathrm{g} / \mathrm{l}-6.5 \mu \mathrm{g} / \mathrm{l}$ ).

\section{Statistics}

Median and interquartile range were calculated to describe continuous variables. Mann Whitney $\mathrm{U}$ and Wilcoxon signed rank tests were used for statistical analysis. Spearman rank correlation coefficients were calculated to describe the association between variables. A p value $<0.05$ was assumed to indicate statistical significance.

\section{Results}

Glycogen phosphorylase isoenzyme BB concentrations in patients with elective coronary artery bypass grafting

Sixteen patients undergoing elective coronary artery bypass grafting had an uneventful perioperative course. All 16 patients had creatine kinase isoenzyme MB catalytic concentrations $<50 \mathrm{U} / 120$ hours after aortic unclamping, and no patient developed new $\mathrm{Q}$ waves perioperatively. None of the 16 patients showed postbypass cardiac failure and all were weaned from extracorporeal circulation without inotropic support (dopamine dosis $<5 \mu \mathrm{g} / \mathrm{kg} \cdot \mathrm{min}$ ). Creatine kinase isoenzyme MB catalytic concentrations in these uneventful patients significantly $(p=0.01)$ increased three hours after aortic unclamping (median peak concentration $18 \mathrm{U} / \mathrm{l}$, range: $4 \mathrm{U} / 1-27 \mathrm{U} / \mathrm{l}$ ) and stayed elevated for 20 hours. Glycogen phosphorylase isoenzyme $\mathrm{BB}$ mass concentrations in these 16 patients significantly increased $(p=0.01)$ after aortic unclamping as well, remained high for 4 hours (median peak concentration $40 \mu \mathrm{g} / \mathrm{l}$, range: 12.7 $\mu \mathrm{g} / 1-47.5 \mu \mathrm{g} / \mathrm{l})$ and decreased to baseline values within 20 hours after aortic unclamping (see fig. 1). In contrast to creatine kinase isoenzyme MB catalytic concentration peaks $(\rho=0.34, p=0.3)$, glycogen phosphorylase isoenzyme BB mass concentration peaks $(\rho=0.59$, $p=0.03$ ) of these 16 patients correlated with aortic crossclamping time.

One patient (patient b, fig. 1) did not fulfil the criteria for perioperative myocardial infarction (creatine kinase isoenzyme $\mathrm{MB}$ catalytic concentration peak $27 \mathrm{U} / 18$ hours after aortic unclamping), but developed cardiac failure in the post cross-clamping period despite prolonged reperfusion on extracorporeal circulation. Perioperative electrocardiographic monitoring for myocardial ischaemia was non-diagnostic in this patient, because of a preexisting left bundle branch block. Preoperative left ventricular function was only mildly impaired (left ventricular enddiastolic pressure: $6 \mathrm{~mm} \mathrm{Hg}$, left ventricular ejection fraction: $46 \%$ ) and the aortic crossclamping time was only 65 minutes. Surgery, however, was complicated by thrombendarterectomy of the left anterior descending coronary artery. The patient could be weaned from extracorporeal circulation only with high dose inotropic support (adrenaline $17 \mu \mathrm{g} / \mathrm{kg} \cdot \mathrm{min}$, amrinone 350 $\mu \mathrm{g} / \mathrm{kg} \cdot \mathrm{min})$. Left ventricular function improved postoperatively and the patient was weaned from catecholamine therapy within 24 hours. In contrast to creatine kinase isoenzyme MB catalytic concentrations, glycogen phosphorylase isoenzyme $\mathrm{BB}$ mass concentrations in this patient (see fig. 1, patient b) were markedly higher than in 16 uneventful cases and exceeded $100 \mu \mathrm{g} / 1$ as early as 1 hour after aortic unclamping, stayed high for 8 hours and decreased to baseline values within 24 hours.

Another patient (patient c, fig. 1) sustained extensive perioperative myocardial infarction (creatine kinase isoenzyme MB peak level $340 \mathrm{U} / 148$ hours after aortic unclamping) and needed intraaortic balloon pumping postoperatively. This patient died from cardiogenic shock on postoperative day 3 and extensive perioperative myocardial infarction was proved by autopsy. Glycogen phosphorylase isoenzyme BB mass concentrations in patient $\mathrm{c}$ steadily increased from aortic unclamping onwards and were markedly higher than in all other patients from 16 hours after aortic unclamping onwards (glycogen phosphorylase isoenzyme BB mäss peak concentration: $2296.3 \mu \mathrm{g} / 148$ hours after aortic unclamping, see fig. 1).

Glycogen phosphorylase isoenzyme BB mass concentrations in 2 patients with emergency coronary artery bypass grafting for evolving myocardial infarction

Both patients were transferred to our institution for emergency coronary artery býpass grafting for evolving 


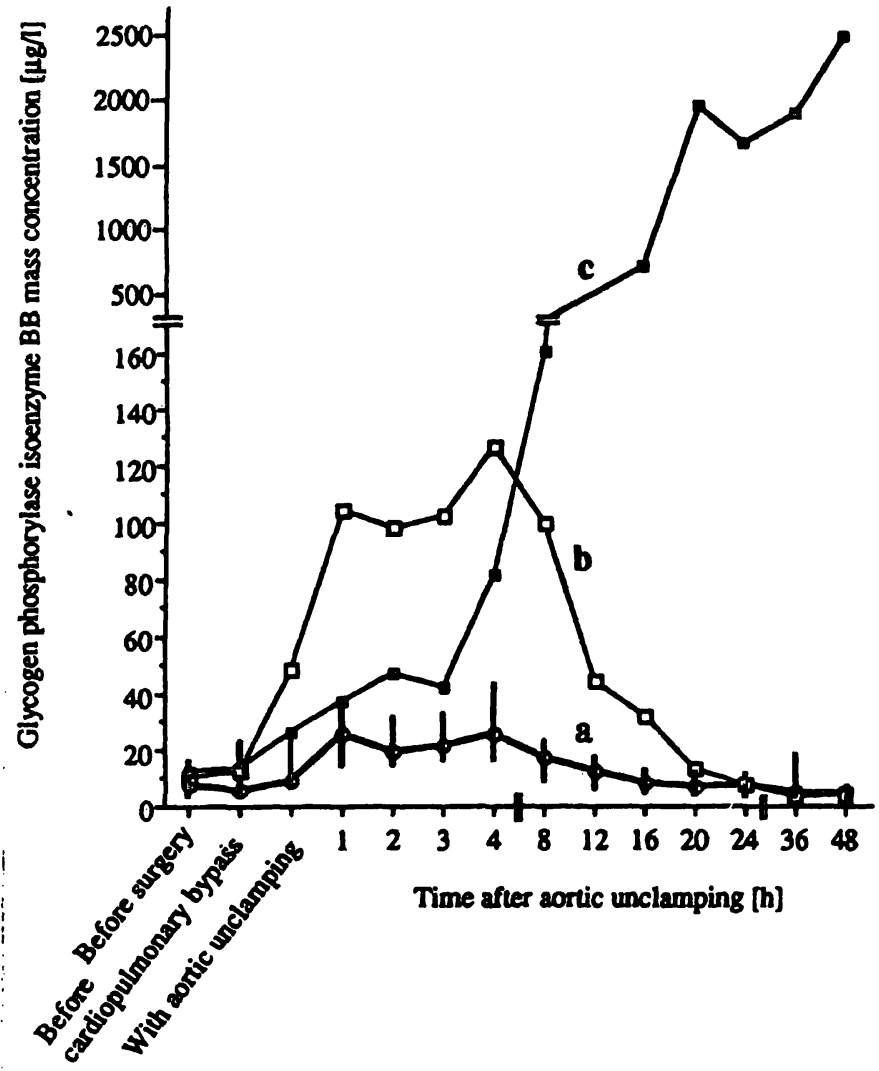

Fig. 1 Glycogen phosphorylase isoenzyme BB mass concentrations in patients with elective coronary artery bypass grafting. a: uneventful patients $(n=16$; data are given as median, bars indicate interquartile range);

b: a patient without perioperative myocardial infarction who sustained postbypass cardiac failure;

c: a patient with extensive perioperative myocardial infarction who died on postoperative day 3 .

myocardial infarction. Both had a left main coronary artery stenosis and a history of angina at rest with ST-T segment changes and increased creatine kinase isoenzyme MB catalytic concentrations. Both patients showed very similar time courses for perioperative creatine kinase isoenzyme MB catalytic concentration (see fig. 2a). Creatine kinase isoenzyme MB catalytic concentrations were already elevated before surgery (creatine kinase isoenzyme MB $25 \mathrm{U} / 1$ and $44 \mathrm{U} / 1$, respectively), staying high perioperatively (creatine kinase isoenzyme MB 23 U/l and $49 \mathrm{U} / \mathrm{l} 20$ hours after aortic unclamping, respectively, see fig. 2a). By contrast, clinical history as well as glycogen phosphorylase isoenzyme BB mass release were markedly different. One patient (patient $D$, fig. 2b) already had elevated glycogen phosphorylase isoenzyme $\mathrm{BB}$ mass concentrations preoperatively (glycogen phosphorylase isoenzyme BB before surgery: $60 \mu \mathrm{g} / \mathrm{l}$ ), but glycogen phosphorylase isoenzyme BB plasma concentrations decreased rapidly after aortic unclamping and were comparable to those in patients with uneventful elective coronary artery bypass grafting (glycogen phosphorylase isoenzyme BB 4 hours after unclamping: $29.3 \mu \mathrm{g} / \mathrm{l}$, glycogen phosphorylase isoenzyme BB 16 hours after unclamping: $14.9 \mu \mathrm{g} / \mathrm{l}$ ). Patient D, complained of repeated and prolonged episodes of angina at rest, which were resistant to intravenous nitroglycerin during the night before surgery. When arriving in the operating theatre patient $D$ was in cardiogenic shock and needed inotropic support with catecholamines. Patient $D$ was easily weaned from the bypass after surgical revascularisation and did well postoperatively. Electrocardiographic monitoring in this patient showed that no new $Q$ waves developed perioperatively. In the other patient (patient $E$, fig. $2 b$ ) glycogen phosphorylase isoenzyme BB mass concentrations were not elevated before surgery $(7.5 \mu \mathrm{g} / \mathrm{l})$. Patient $E$ showed a marked, persistent glycogen phosphorylase isoenzyme BB increase (peak concentration: $414 \mu \mathrm{g} / \mathrm{l}$ ) from 8 hours after unclamping until postoperative day 2 . This patient did not complain of angina during the immediate preoperative period and arrived in the operating theatre haemodynamically stable. Patient E developed severe cardiac failure after aortic unclamping and could be weaned from cardiopulmonary bypass only by admin-
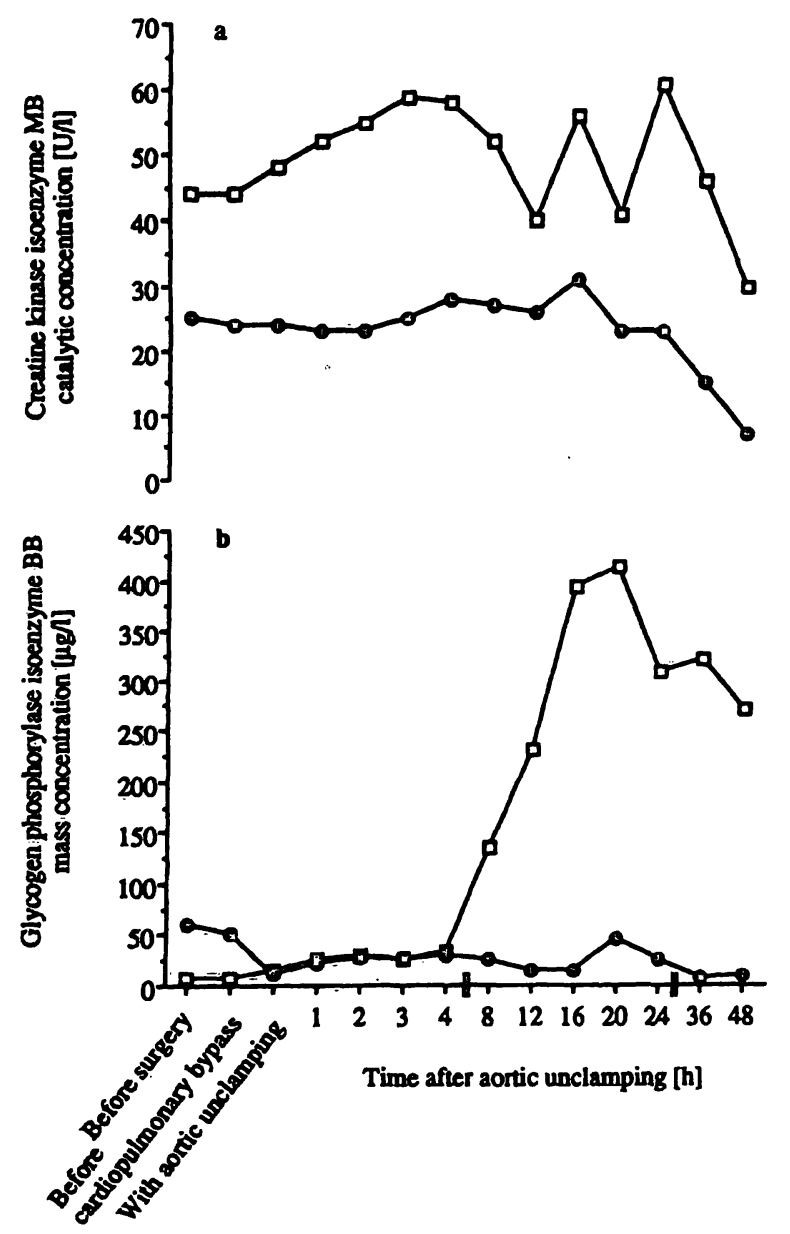

Fig. 2 Time course of creatine kinase isoenzyme MB and glycogen phosphorylase isoenzyme $\mathrm{BB}$ in 2 patients with emergency coronary artery bypass grafting for evolving myocardial infarction. - - patient (patient D): preoperative cardiogenic shock and uneventful postoperative clinical course.

$-\square-$ patient (patient E): stable haemodynamics preoperatively and postbypass cardiac failure. 
istration of high doses of positive inotropic agents (adrenaline $14 \mu \mathrm{g} / \mathrm{kg} \cdot \min$, amrinone $350 \mu \mathrm{g} / \mathrm{kg} \cdot \min$ ). This patient had a prolonged low cardiac output syndrome postoperatively. Electrocardiographic monitoring in this patient demonstrated the development of a new left bundle branch block perioperatively.

\section{Discussion}

Creatine kinase isoenzyme $\mathrm{MB}$ catalytic concentration is of limited sensitivity and specificity in the laboratory diagnosis of perioperative myocardial ischaemia and small perioperative myocardial infarctions $(8,9)$. Creatine kinase isoenzyme $\mathrm{MB}$ catalytic concentration also does not meet current needs for a highly sensitive marker of minor myocardial injury, which is necessary to compare different means of cardioprotection (10). Preliminary data indicate that catalytic concentrations of glycogen phosphorylase and glycogen phosphorylase isoenzyme' $\mathrm{BB}$ (immunoinhibition by polyclonal antibodies) are sensitive laboratory markers of perioperative myocardial ischaemia in patients undergoing coronary artery bypass grafting $(11,12)$. Therefore, we expected glycogen phosphorylase isoenzyme BB mass concentration to offer advantages over creatine kinase isoenzyme MB catalytic concentration in the evaluation of perioperative myocardial injury. In fact, glycogen phosphorylase isoenzyme $\mathrm{BB}$ mass peak concentrations, but not creatine kinase isoenzyme MB catalytic concentration peaks correlated significantly with aortic crossclamping time. Furthermore, in a patient with cardiac failure after aortic unclamping, who did not fulfill criteria for perioperative myocardial infarction, glycogen phosphorylase isoenzyme $\mathrm{BB}$ mass concentrations, but not creatine kinase isoenzyme $\mathrm{MB}$ catalytic concentrations were markedly higher than in uneventful cases. Electrocardiography was non-diagnostic in this patient, due to a preexisting left bundle branch block, and could not be used to support the diagnosis of myocardial injury, which was based on clinical presentation. Nevertheless, ischaemic myocardial injury not detected by creatine kinase isoenzyme MB catalytic concentration is the most likely explanation for postbypass cardiac failure in this patient who had an only mildly reduced preoperative left ventricular ejection fraction and an aortic crossclamping time of 65 minutes. The advantages of glycogen phosphorylase isoenzyme BB in the evaluation of perioperative myocardial injury can be explained at least partly by the higher relative increase of glycogen phosphorylase isoenzyme BB compared with creatine kinase isoenzyme $M B$ which was observed in patients with acute myocardial infarction (5). Due to their higher relative increase, glycogen phosphorylase isoenzyme BB plasma concentrations can be elevated considerably even after limited myocardial necrosis, which does not necessarily result in a detectable elevation in creatine kinase isoenzyme MB catalytic concentration. On the other hand, these findings might also support the hypothesis that glycogen phosphorylase isoenzyme BB is released from ischaemic but still viable myòtardium, which does not necessarily release other myocardial marker proteins. In cardiomyocytes, glycogen phosphorylase isoenzyme BB exists in association with glycogen, and the sarcoplasmatic reticulum forms the sarcoplasmatic reticulum-glycogenolysis-complex (13). During myocardial ischaemia this complex is broken down (13). Glycogen phosphorylase isoenzyme $\mathrm{BB}$ is released into the sarcoplasma, which results in a large soluble cytosolic glycogen phosphorylase isoenzyme $\mathrm{BB}$ pool, and a high glycogen phosphorylase isoenzyme BB concentration gradient between the sarcoplasma and the extracellular space. In case of severe, but still reversible ischaemic injury of the moyocardium, plasma membrane permeability is also altered and soluble proteins may diffuse out of myocytes and appear in the plasma $(3,14,15)$.

Perioperative glycogen phosphorylase isoenzyme $\mathrm{BB}$ mass, but not perioperative creatine kinase isoenzyme MB catalytic activity release correlated well with clinical evidence of myocardial ischaemia in 2 patients with emergency coronary artery bypass grafting for evolving myocardial infarction. Glycogen phosphorylase isoenzyme BB plasma half life (4 to 6 hours; unpublished data) is markedly shorter than creatine kinase isoenzyme MB plasma half life (12 to 16 hours) (16). Therefore, due to the very low $K_{\mathrm{d}}$-value (12), glycogen phosphorylase isoenzyme BB plasma concentrations will return to normal earlier than creatine kinase isoenzyme MB catalytic concentrations in patients with myocardial infarction without ongoing ischaemia. Glycogen phosphorylase isoenzyme BB measurements will, therefore, identify patients with successful surgical revascularisation earlier and more reliable than creatine kinase isoenzyme MB determinations. Furthermore, glycogen phosphorylase isoenzyme BB mass concentration increases earlier than creatine kinase isoenzyme $M B$ catalytic concentration in patients with acute myocardial infarction (5). The differences in release kinetics and the possible release of glycogen phosphorylase from ischaemic but still viable myocardium might explain the advantages of glycogen phosphorylase isoenzyme BB over creatine kinase isoenzyme MB in the laboratory diagnosis of additional perioperative myocardial injury in patients undergoing emergency bypass surgery.

All 3 patients in whom glycogen phosphorylase isoenzyme $\mathrm{BB}$, but not creatine kinase isoenzyme MB, indicated myocardial injury received catecholamine therapy. Experimental results indicate that without concomitant 
cell membrane damage, even high doses of adrenaline do not cause a detectable glycogen phosphorylase release from cardiomyocytes (17). Therefore, it is unlikely that catecholamine therapy alone, in the absence of additional myocardial injury, caused the glycogen phosphorylase release observed in these 3 patients. Only isoenzyme MM of glycogen phosphorylase has been isolated from skeletal muscle (1). Cross-reaction of the glycogen phosphorylase isoenzyme $\mathrm{BB}$ assay with $\mathrm{MM}$ and $\mathrm{LL}$ isoenzyme was below $1 \%$ (7). Therefore, skeletal muscle injury and liver damage do not contribute to the perioperative glycogen phosphorylase isoenzyme BB increase observed in our patients. Haemoglobin and bilirubin do not interfere with the glycogen phosphorylase isoenzyme BB assay used (7). Except for the patient with perioperative myocardial infarction and cardiogenic shock, who became anuric a few hours before death (postoperative day 3), no patient sustained perioperative renal failure. In addition to the human myocar-

\section{References}

1. Will, H., Krause, E. G., Boehm, M., Guski, H. \& Wollenberger, A. (1974) Kinetische Eigenschaften der Isoenzyme der Glykogenphosphorylase b aus Herz- und Skelettmuskulatur des Menschen. Acta Biol. Med. Germ. 33, 149-160.

2. Krause, E. G., Will, H., Boehm, M. \& Wollenberger, A. (1975) The assay of glycogen phosphorylase in human blood serum and its application to the diagnosis of myocardial infarction. Clin. Chim. Acta 58, 145-154.

3. Michael, L. H., Hunt, J. R., Weilbaecher, D., Perryman, M. B., Roberts, R., Lewis, R. M. \& Entman, M. L. (1985) Creatine kinase and phosphorylase in cardiac lymph: Coronary occlusion and reperfusion. Am. J. Physiol. 248, 350-359.

4. Rabitzsch, G., Schulz, H., Onnen, K., Koessler, A. \& Krause, E. G. (1987) Immunoinhibition assay of the serum activity of human glycogen isophosphorylase $\mathrm{BB}$ in the diagnosis of acute myocardial ischaemia. Biomed. Biochim. Acta 46, S584-S588.

5. Rabitzsch, G., Mair, J., Leichleitner, P., Noll, F., Hofmann, U., Krause, E. G., Dienstl, F. \& Puschendorf, B. (1993) Isoenzyme BB of glycogenphosphorylase $b$ and myocardial infarction (letter). Lancet 341, 1032-1033.

6. Graeber, G., Shawl, F., Head, H., Wolf, R. E., Burge, J. R., Cafferty, P. J., Lough, F. C. \& Zajtchuk, R. (1986) Changes in serum creatine kinase and lactate dehydrogenase caused by acute perioperative myocardial infarction and by transatrial cardiac surgical procedures. J. Thorac. Cardiovasc. Surg. 92, $63-72$.

7. Hofmann, U., Rabitzsch, G., Loester, K., Handschack, W., Noll, F. \& Krause, E.-G. (1989) Immunoenzymometric assay for the heart specific glycogen phosphorylase $B B$ in human serum using monoclonal antibodies. Biomed. Biochim. Acta $48, \mathrm{~S} 132-\mathrm{S} 136$.

8. Roberts, A. J. (1983) Perioperative myocardial infarction and changes in left ventricular performance related to coronary artery bypass graft surgery. Ann. Thor. Surg. 35, 208-225.

9. Haagensen, R. \& Steen, P. A. (1988) Perioperative myocardial infarction. Br. J. Anaesth. 61, 24-37.

10. Seguin, J. R., Saussine, M., Ferriere, M., Leger, J. J., Leger, J., Larue, C., Calzolari, C., Grolleau, R. \& Chaptal, P. A. (1989) Myosin: A highly sensitive indicator of myocardial ne- dium, significant amounts of glycogen phosphorylase isoenzyme BB are also found in the brain (18). Significant cerebral injury as a source of increased glycogen phosphorylase isoenzyme BB plasma concentrations could be excluded with certainty in all patients. Therefore, we conclude that the differences in glycogen phosphorylase isoenzyme BB plasma concentrations observed in these 20 patients with coronary artery bypass grafting reflect differences in the extent of myocardial injury.

In summary, our results indicate that glycogen phosphorylase isoenzyme BB mass concentration is a very sensitive marker of perioperative myocardial injury in patients undergoing coronary artery bypass grafting. Glycogen phosphorylase isoenzyme $\mathrm{BB}$ mass measurements may be particularly useful in the perioperative laboratory monitoring of myocardial ischaemia in patients undergoing emergency coronary artery bypass grafting.

crosis after cardiac operations. J. Thorac. Cardiovasc. Surg. 98 , 397-401.

11. Rabitzsch, G., Goos, H., Noehring, J., Lembcke, J., Lindenau, K.-F., Krause, E.-G., Parsi, R.-A. \& Heine, H. (1984) Glycogen phosphorylase $\mathrm{b}$ activity in human serum after acute myocardial infarction and coronary bypass surgery. In: Cellular and Molecular Aspects of the Regulation of the Heart, Abh. Akad. Wiss. DDR, Akad. Verlag, Berlin 1984, IN; pp. $251-$ 253.

12. Kuntze, T., Rabitzsch, G., Hofman, U., Lindenau, K.-F. \& Krause, E.-G. (1991) Release of the isoenzyme BB of glycogen phosphorylase $\mathrm{b}$ and detection of perioperative infarction in patients undergoing aorto-coronary bypass surgery (abstract). Eur. Surg. Res. 23, S1, 105.

13. Entman, M. L., Kaniike, K., Goldstein, M. A., Nelson, T. E., Bornet, E. P., Futch, T. W. \& Schwartz, A. (1976) Association of glycogenolysis with cardiac sarcoplasmic reticulum. J. Biol. Chem. 251, 3140-3146.

14. Krause, E. G., Haertwig, A. \& Rabitzsch, G. (1989) On the release of glycogen phosphorylase from heart muscle: Effect of substrate depletion, ischaemia and of imipramine. Biomed. Biochim. Acta 48, S77-S82.

15. Piper, H. M., Schwartz, P., Spahr, P., Hütter, J. F. \& Spieckermann, P. G. (1984) Early enzyme release from myocardial cells is not due to irreversible cell damage. J. Mol. Cell. Cardiol. $16,385-388$.

16. Jones, M. G. \& Swarimathon, R. (1990) The clinical biochemistry of creatine kinase. J. Int. Fed. Clin. Chem. 2, 108-114.

17. Schulze, W., Krause, E. G. \& Wollenberger, A. (1971) On the fate of glycogen phosphorylase in the ischemic and infarcting myocardium. J. Mol. Cell. Cardiol. 2, 241-251.

18. Newgard, C. B., Littmann, D. R., van Genderen, C., Smith, M. \& Fletterick, R. J. (1988) Human brain glycogen phosphorylase. J. Biol. Chem. 263, 3850-3857.

Prof. Dr. Bernd Puschendorf Institut für Medizinische Chemie und Biochemie Fritz-Pregl-Straße 3 A-6020 Innsbruck Austria 
